\title{
Heparin induced bullous hemorrhagic dermatosis at a site distant from the injection. A report of five cases*
}

\author{
Vanessa Gargallo ${ }^{1}$ \\ José Luis Rodríguez-Peralto ${ }^{1}$
}

\author{
Fatima Tous Romero ${ }^{1}$ \\ Carlos Zarco ${ }^{1}$
}

Heparins both unfractionated and low-molecular-weight are associated with some cutaneous complications including hematomas, ecchymosis, erythematous plaques, nodules, skin necrosis, contact dermatitis and urticaria, all occurring more commonly at local subcutaneous injection sites. ${ }^{1,2}$ First reported at 2006 by Perrinaud et al, bullous hemorrhagic dermatosis is a rare cutaneous reaction to heparin in which hemorrhagic intraepidermal bullae appear in areas distant from the heparin injection sites and of which there are less than 20 cases described in the literature. ${ }^{1,2}$

We present 5 cases of heparin induced bullous hemorrhagic dermatosis at a site distant from the injection. The characteristics of each patient are detailed in table 1 . All the patients were male with mean age of 74 years, in treatment with enoxaparin at different doses. Patients 3 and 4 were also taking antiplatelet drugs. The onset of bullae was 8-20 days after the beginning of the heparin therapy and the lesions were asymptomatic in all cases. Biopsy was performed in the 5 cases, showing intraepidermic blister filled with red blood cells, without any signs of vasculitis or vessels thrombosis, and heparin-induced bullous hemorrhagic dermatoses was diagnosed (Figure 1A). Laboratory tests' results, blood count and coagulation studies were normal. Patients 2, 3, and 4 had pruritic conditions pre- vious to the onset of lesions; therefore they scratched their skin. We observed that these patients presented more lesions and that they were more disseminated than in those patients without pruritus. What is more relevant, in patients 2, 3 and 4 some of the lesions had a linear, Koebner-like, arrangement (Figure 1B). Strikingly, patient 3 developed new lesions on the stitches at the site of biopsy (Figure 1C). Patient 5 had no pruritic condition, but the appearance of the lesions was clearly associated to an occasional scratch on the area. In three of our five cases we maintained the treatment; two of them self-resolved without discontinuation but treatment was changed in patient 2 because new lesions kept appearing, but it also had a complete resolution within few weeks. The reaction to heparin seemed to be retarded as proved by the late onset of the bullae, ranging from 8 to 20 days after the beginning of the heparin therapy. This data is also consistent with the reports previously published. ${ }^{1,3}$

The pathogenesis of this condition is unclear. Since some patients were receiving high doses of heparin it has been proposed a dose-related reaction. ${ }^{3}$ In our series only one patient received very high doses of heparin, and two patients received low dose. Other authors also agree with this observation, ${ }^{4}$ being unlikely an overdose phenomenon. A synergic mechanism has also been proposed for

Received on 23.11.2015

Approved by the Advisory Board and accepted for publication on 18.06.2016

* Study performed at the Department of Dermatology, 12 de Octubre University Hospital, Madrid, Spain. Financial Support: None.

Conflict of Interest: None.

12 de Octubre University Hospital - Madrid, Spain.

(C)2016 by Anais Brasileiros de Dermatologia 


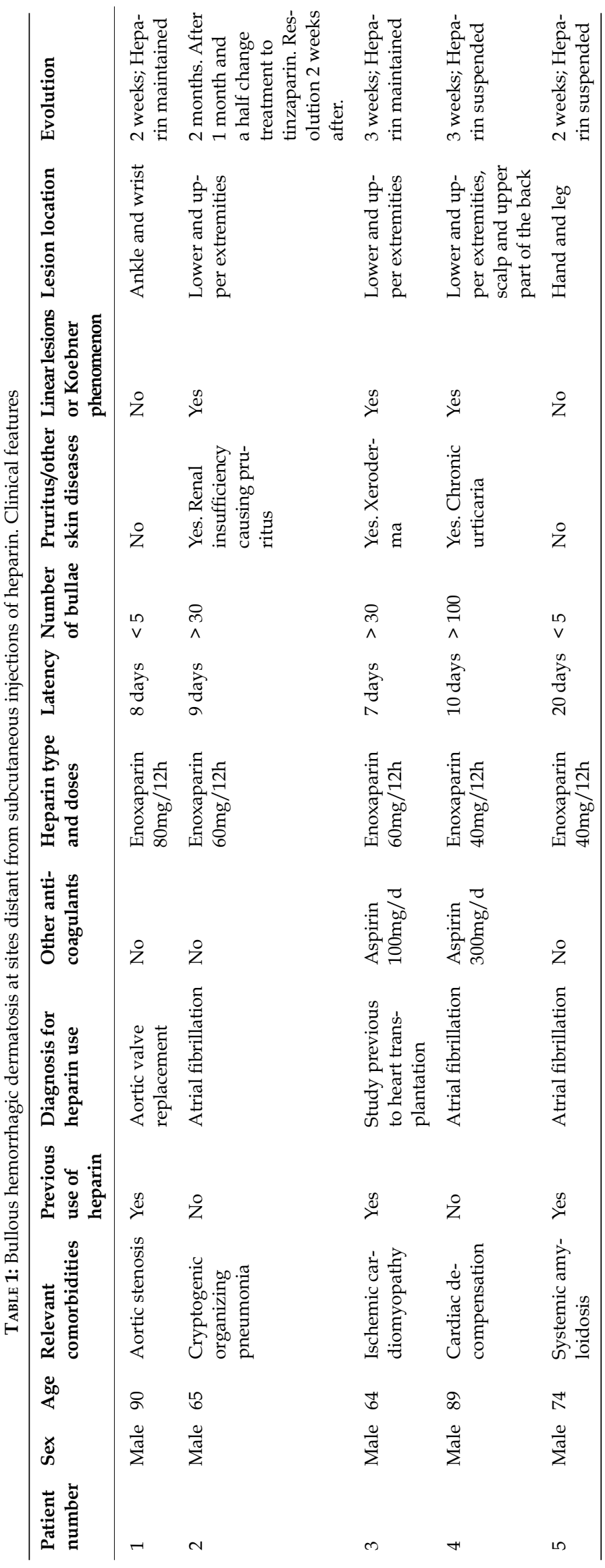

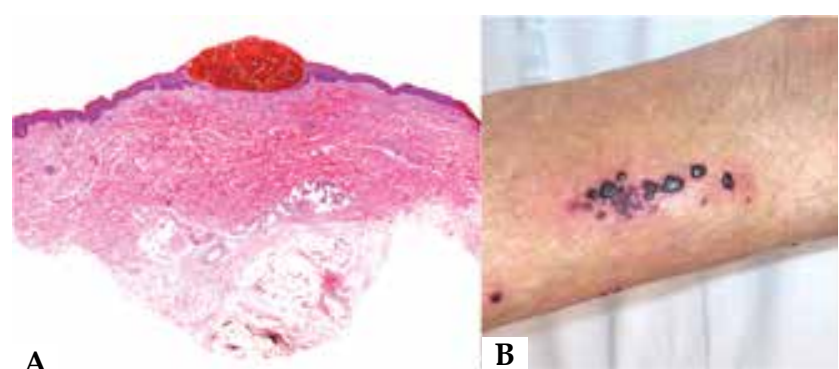

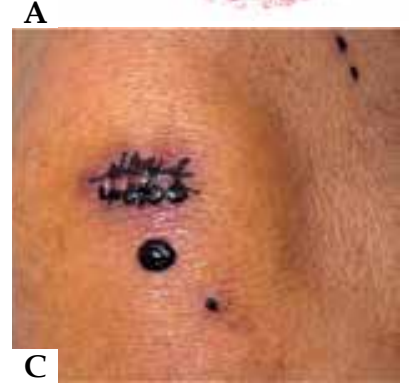

Figure 1: Bullous hemorrhagic dermatosis at sites distant from subcutaneous injections of heparin. A: Histopathologic findings: subepidermal blister filled with red blood cells. Case 1. B: Linear arrangement of the lesions. Case 4. C: Koebner phenomenon with development of new lesions on the area in which the stiches were given. Case 3

patients treated with one or more anticoagulants or antiplatelet drugs, however, cases also occurred without anticoagulants and with normal coagulation studies. ${ }^{1,3,4}$ Only two of our patients were taking anticoagulants and coagulation studies were normal in all the cases, therefore we doubt the contribution of theses factors in the development of the bullae. Hypersensitivity reaction to heparin injection has been suggested ${ }^{4}$ but the absence of eosinophils on histology does not support this theory. Previous reports also show lesions arranged in groups on small skin areas ${ }^{5}$ or showing a linear, Koebner-like, arrangement. ${ }^{1,4}$ This consistent with the relevance of an external trauma causing or increasing the number of lesions. More recent reports indicate that in most cases discontinuation of the treatment is not necessary. ${ }^{2,4}$ In the cases in which we decided to maintain treatment the lesions eventually disappeared. However, in one case the lesions where persistent for a month and a half hence treatment was changed with complete resolution afterwards. Therefore if the appearance of new lesions continues for longer than three weeks it should be advisable to change the anticoagulant therapy. Our observations prove an increased number of lesions after trauma. This might not be the only cause but it is for sure an important factor in the development of the bullae, with a significant increase in the number of lesions in patients suffering from pruritic conditions. In fact, disseminated lesions emerged only in these patients. Since Koebner phenomenon occurs in this disease dermatologist should be aware that there can be disseminated and more persistent lesions in patients with pruritic conditions. An individualized approach, taking into account the extension, time of evolution and the importance of anticoagulation in the context of each patient helps to decide whether to suspend, maintain or change therapy.] 


\section{REFERENCES}

1. Perrinaud A, Jacobi D, Machet MC, Grodet C, Gruel Y, Machet L. Bullous hemorrhagic dermatosis occurring at sites distant from subcutaneous injections of heparin: Three cases. J Am Acad Dermatol. 2006;54:S5-7.

2. Öztürk S, Can I, Erden I, Akyol H, Solmaz OA. Enoxaparin-induced hemorrhagic bullous dermatosis in a leprosy patient. Cutan Ocul Toxicol. 2015;34(3):254-6.

3. Maldonado Cid P, Moreno Alonso de Celada R, Herranz Pinto P, Noguera Morel L, Feltes Ochoa R, Beato Merino MJ, et al. Bullous hemorrhagic dermatosis at sites distant from subcutaneous injections of heparin: A report of 5 cases $\mathrm{J}$ Am Acad Dermatol. 2012;67:e220-2.

4. Peña ZG, Suszko JW, Morrison LH. Hemorrhagic bullae in a 73-year-old man. Bullous hemorrhagic dermatosis related to enoxaparin use. JAMA Dermatol. 2013 Jul;149(7):871-2

5. Beltraminelli H, Itin P, Cerroni L. Intraepidermal bullous haemorrhage during anticoagulation with low-molecular-weight heparin: two cases. $\mathrm{Br} \mathrm{J}$ Dermatol. 2009;161:191-3.

\author{
MAILING ADDRESS: \\ Vanessa Gargallo \\ Avda de Córdoba s/n \\ Madrid \\ 28232 Spain. \\ Email:vgmoneva@gmail.com
}

How to cite this article: Gargallo V, Romero Tous Romero F, Rodríguez-Peralto JL, Zarco C. Heparin induced bullous hemorrhagic dermatosis at a site distant from the injection. A report of five cases. An Bras Dermatol. 2016;91(6):857-9. 